\title{
JHH Young Investigator Award 2019: interview with the winner Swaib Lule
}

\author{
Swaib A. Lule $\mathbb{1}^{1,2}$
}

Received: 16 June 2020 / Revised: 29 June 2020 / Accepted: 21 July 2020 / Published online: 3 August 2020

(c) Springer Nature Limited 2020

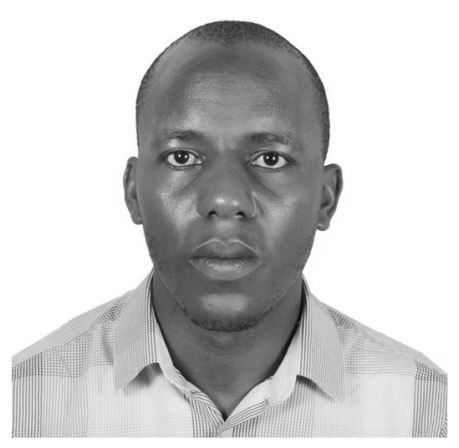

\section{Could you tell us something about yourself and your unit?}

This work was part of my Ph.D. at the London School of Hygiene and Tropical Medicine (LSHTM) supervised by Dr. Emily Webb, Prof. Liam Smeeth and Prof. Alison Elliott. I am grateful to Prof. Elliott for the opportunity to use the Entebbe Mother and Baby Study (EMaBS) birth cohort to investigate the impact of early-life, life-course and genetic factors on blood pressure (BP) among young Africans. Previously, I worked as a Research Scientist with the EMaBS, an ongoing prospective study of 2507 mother-baby pairs, in the Immunomodulation and Vaccines Programme at the MRC/UVRI and LSHTM Uganda Research Unit in Entebbe, Uganda.

This birth cohort was established in 2003 as a deworming trial to investigate the effects of worms and their treatment in

Swaib A. Lule

swaiblule@yahoo.com

1 Institute for Global Health, University College London (UCL), London, UK

2 Immunomodulation and Vaccines Programme, MRC/UVRI \& LSHTM Uganda Research Unit, Entebbe, Uganda pregnancy and childhood on response to childhood vaccines and infections. The trial phase ended in 2011 when all participants were past the age of 5 years. In 2014, we designed a sub-study among 10- and 11-year-old participants who were still in active follow-up to investigate early-life, life-course and genetic factors associated with BP in early adolescents.

The cohort has mainly been supported through various Senior Wellcome Trust fellowships with supplementary funding from the Medical Research Council (MRC) to study cardiovascular diseases (CVDs) risk factors in the EMaBS cohort. My Ph.D. was funded by the Commonwealth Scholarship Commission.

\section{Why did you undertake this research?}

Africa is a continent with an increasing burden of noncommunicable diseases, a high prevalence of low birth weight infants, infectious diseases and malnutrition, and high genetic diversity. The relationships between these factors and BP are unknown and understudied on the continent. The existence of the EMaBS, a large birth cohort with longitudinally collected data on early-life and lifecourse factors (such as birth weight, obesity and childhood infections) was an opportunity to understand the role of these factors in programming BP later in life.

\section{What did you learn from this research?}

Our systematic review showed limited evidence from Africa on the relationship between early-life factors (in particular birth weight) and BP among young Africans. From the few publications from Africa, this relationship varied greatly with participants' age. Our study showed that infants' postnatal weight gain was an important factor in programming later BP, with fast-growing low birth weight individuals particularly affected. We also showed that maternal body mass index (BMI) and higher education status during pregnancy and 
adolescents' age, waist circumference, BMI, family history of hypertension and current Trichuris infection were positively associated with higher BP in adolescents. Childhood malaria infections, higher vegetable and fruit consumption were associated with lower BP in these adolescents.

\section{Could you expand on the significance of your findings?}

Our findings suggest that infectious diseases such as malaria and Trichuris could be important in the programming of BP later in life. The role of these and other infections in programming non-communicable diseases later in life need further studying.

Adopting a life-course approach could be vital in the prevention and control of BP later in life. Interventions initiated early in life targeting those who were small at birth, with a family history of hypertension, aiming to reduce adiposity (in pregnancy and or adolescence) and promoting fruit and vegetable consumption might contribute to reducing the risk of later high BP and subsequent CVDs. Mores studies from Africa on the role of early-life and life-course in programming BP later in life are needed.

\section{Can you tell us about any research you're currently undertaking that is related to the paper?}

Currently, we are investigating the lipid profiles of the participants in the EMaBS. We are also describing the distribution of BP among children and young adolescents from rural southwestern Uganda and hope to understand factors associated with elevated BP in this rural population.

\section{Compliance with ethical standards}

Conflict of interest The author declares that he has no conflict of interest.

Publisher's note Springer Nature remains neutral with regard to jurisdictional claims in published maps and institutional affiliations. 